Elsevier required licence: (C) $<2020>$. This manuscript version is made available under the CC-BY-NCND 4.0 license http://creativecommons.org/licenses/by-nc-nd/4.0/

The definitive publisher version is available online at

[https://www.sciencedirect.com/science/article/pii/S0022460X2030482X?via\%3Dihub] 


\title{
Non-Negative Intensity for Planar Structures Under Stochastic Excitation
}

\author{
M. Karimi ${ }^{*}$, L. Maxit ${ }^{2}$, V. Meyer ${ }^{3}$, S. Marburg ${ }^{4}$, R. Kirby ${ }^{1}$ \\ ${ }^{1}$ Centre for Audio, Acoustics and Vibration, University of Technology Sydney, Sydney, \\ Australia \\ ${ }^{2}$ Univ Lyon, INSA-Lyon, Laboratoire Vibrations-Acoustique (LVA), 25 bis, av. Jean \\ Capelle, F-69621, Villeurbanne Cedex, France \\ ${ }^{3}$ Naval Group Research, 199 avenue Pierre-Gilles de Gennes, 83190 Ollioules, France \\ 4 Chair of Vibroacoustics of Vehicles and Machines, Department of Mechanical \\ Engineering, Technische Universität München, München, Germany
}

\begin{abstract}
Identification of regions on a vibrating structure which radiate energy to the far field is critical in many areas of engineering. Non-negative intensity is a means to visualize contributions of local surface regions to sound power from vibrating structures. Whilst the non-negative intensity has been used for structures under deterministic excitation due to structural forces or harmonic incident acoustic pressure excitation, it has not been considered for analyzing a structure under stochastic excitation. This work analytically formulates non-negative intensity in the wavenumber domain to investigate the surface areas on a vibrating planar structure that are contributing to the radiated sound power in the far field. The non-negative intensity is derived in terms of the cross spectrum density function of the stochastic field and the sensitivity functions of either the acoustic pressure or normal fluid particle velocity. The proposed formulation can be used for both infinite planar structure and finite plate in an infinite baffle. To demonstrate the technique, a simply supported baffled panel excited by a turbulent boundary layer as well as an acoustic diffuse field is considered and those regions contributing to the radiated sound power are identified. It is demonstrated that the nonnegative intensity distribution is dependent on the stochastic excitation. It is also found that for a panel under stochastic excitation the more the nonnegative intensity distribution is concentrated within the panel surface, the
\end{abstract}

\footnotetext{
${ }^{*}$ Corresponding author

Email address: Mahmoud.karimi@uts.edu.au
}

Preprint submitted to Journal of Sound and Vibration

August 30, 2020 
more efficient the panel radiates sound to the far field.

Keywords: Acoustic radiation, non-negative intensity, surface contribution, stochastic excitation, turbulent boundary layer, acoustic diffuse field

\section{INTRODUCTION}

Reconstruction techniques of sound sources such as near-field acoustic holography (NAH), inverse boundary element method (BEM) and the equivalent sources methods are widely used in industry 1. In many engineering applications, it is important to identify the regions on a vibrating structure which radiate energy to the far field. This identification can help design engineers to gain a deeper understanding about the noise generation mechanism, and it also allows targeted mitigation strategies to be explored. For example, noise reduction can be achieved by modifying geometry and structural properties. Acoustic intensity can help with identifying hot spots on the structure. However, intensity is usually highly bipolar and has positive and negative values that correspond to energy sources and sinks on the surface of the radiating structure. Therefore, the near-field cancellation effects occur when integrating the positive and negative components of the normal acoustic intensity over the surface of the structure. Williams [2, 3 introduced the supersonic intensity (SSI) formulation in the wavenumber domain. The SSI was employed to locate the areas on the source surface which effectively contribute to the far-field pressure. The SSI eliminates the contribution to the pressure and the velocity on the source of the high wavenumber components (subsonic components), which are evanescent and do not contribute to the far field. The modified velocity and pressure obtained by considering only the wavenumber in the acoustic circle were termed supersonic velocity and supersonic pressure respectively.

The SSI was computed in the space domain using a two-dimensional convolution between the acoustic field and a spatial filter mask by FernandezGrande et al. [4]. The filter corresponds to the space domain representation of the acoustic circle. Hence, only the acoustic waves that propagate effectively to the far field were taken into account. The numerical technique was validated by an experimental study on planar radiators. Fernandez-Grande and Jacobsen [5] quantitatively examined the accuracy of the supersonic intensity. They quantified the error introduced by the finite measurement aperture. It was demonstrated that the error was substantial at low frequen- 
cies. The study showed that using an extended aperture and/or an increased cut-off frequency the error can be diminished. Valdivia et al. [6] employed supersonic acoustic intensity to locate radiating regions on a vibrating structure of arbitrarily shaped geometries. They removed the evanescent waves from the NAH measurement. A method based on a stable invertible representation of the radiated power operator was proposed. The stable invertible operator was derived using the equivalent source formulation and a complete spectral basis. The proposed method was validated using experimental data from a vibrating ship-hull structure.

Magalhães and Tenenbaum [7] extended the SSI technique to consider arbitrarily shaped sources. Their work was based on the BEM and singular value decomposition. Marburg et al. 8 formulated the non-negative intensity (NNI) using the BEM to identify the surface areas of a vibrating structure that contribute to the radiated sound power. The acoustic radiation modes were employed to compute the surface contributions of the structure for all boundaries of the acoustic domain. Williams [9] proposed two analytical formulae for the NNI based on the pressure and normal fluid particle velocity for planar structures under deterministic excitation. It was shown that both formulae yield almost identical results in prediction of the regions of a structure that emit sound to the far field.

Junior and Tenenbaum [10] proposed an equivalent technique to the SSI based on the BEM called useful intensity. The technique does not require the construction of a hologram to evaluate the acoustic pressure from the known normal velocity field on the vibrating surface. Both the analytical SSI and the numerical useful intensity methods were used by Ferreira et al. [11 to examine the sound radiated from rectangular baffled panels. Eight different combinations of classical boundary conditions were considered. It was shown that the results obtained using the useful intensity were not strictly the same as those obtained using the SSI. The NNI based on the BEM was also employed to identify the surface areas of a rigid sphere and a rigid cylinder that contributes to the scattered sound power [12]. The same technique was applied to localize the surface areas of vibrating structure to radiated sound power [13; 14]. The surface contribution from a panel to the radiated sound power for different modes was numerically investigated [14]. The numerical results were validated by NAH measurements. Similar distributions of numerical and experimental NNI were observed at each mode. Liu et al. [15] used the NNI based on the BEM to investigate the effect of inhomogeneous Rayleigh damping on the surface contributions to radiated sound 
power. It was found that traveling waves propagate to the regions with higher damping. Wilkes et al. [16] applied the NNI method to a fluid-loaded steel spherical shell excited by a point/ring force. A hybrid finite element and fast multipole boundary element method (FMBEM) was used to solve the structural-acoustic problem. The boundary field was then used in the FMBEM solver to compute the NNI.

Identification of source velocities on 3D structures in non-anechoic environments using the inverse patch transfer functions (IPTF) method was first introduced by Aucejo et al. [17. The direct patch transfer functions method can be used to predict the structural velocity or the sound pressure of a domain containing acoustic sources by calculating acoustic impedances of uncoupled sub-domains. The IPTF method can identify the unknown sources by measuring the coupling velocity at an arbitrarily defined surface surrounding the source. Vigoureux et al. [18] investigated rigorous criteria needed to obtain accurate results using IPTF to identify sources in a non-anechoic or reverberant environment on an irregularly shaped structure. Further, a procedure was proposed to compute intensity of the source and wall pressure without any additional measurement. A frequency band was detected for which the IPTF method was not providing accurate results. This was attributed to the presence of evanescent waves. Valdivia [19; 20] developed a method based on the spectral decomposition of the power operator that yielded an NNI expression to efficiently compute the supersonic components from acoustic pressure measurements for arbitrary geometries. Using numerical models it was shown that the proposed NNI matched the SSI.

Stochastic excitations such as turbulent boundary layer (TBL) and acoustic diffuse field (ADF) are widely encountered in transportation systems 2123]. For example, aircraft, satellite, marine vessels, high speed trains and cars are subject to random and non-deterministic excitations throughout their operations. While surface contribution techniques such as the SSI and NNI have been developed for structures under deterministic excitation, they have not been applied for analyzing a structure under stochastic excitation. In this work, the NNI is analytically formulated for planar structures under stochastic excitation in the wavenumber domain. The proposed formulation is valid for both infinite planar structure and finite plate in an infinite baffle. Two formulae are developed for the NNI which are in terms of the cross spectrum density function of the stochastic field and the sensitivity functions of either the acoustic pressure or normal fluid particle velocity. The technique 
is implemented to identify the regions of a vibrating simply supported baffled panel contributing to the radiated sound power. Both TBL and ADF excitations are considered to illustrate the proposed technique.

\section{Radiated Acoustic Power}

The radiated acoustic power of an infinite planar structure or a finite plate in an infinite baffle under stochastic excitation can be obtained by integrating the normal active intensity $I_{\text {act }}$, corresponding to the cross spectrum between the sound pressure and the normal fluid particle velocity denoted by $S_{p v_{f}}$, over the infinite boundary surface as follows [24; 25]

$$
\Pi_{\text {rad }}(\omega)=\int_{\infty} I_{\text {act }} \mathrm{d} \mathbf{x}=\int_{\infty} \operatorname{Re}\left\{S_{p v_{f}}(\mathbf{x}, \omega)\right\} \mathrm{d} \mathbf{x},
$$

where $\mathbf{x}=(x, y)$, and $\omega$ is the angular frequency. The cross spectrum is given by the following analytical expression [25]

$$
S_{p v_{f}}(\mathbf{x}, \omega)=\frac{1}{4 \pi^{2}} \int_{\infty} H_{p}(\mathbf{x}, \mathbf{k}, \omega) H_{v}^{*}(\mathbf{x}, \mathbf{k}, \omega) \phi_{p p}(\mathbf{k}, \omega) \mathrm{d} \mathbf{k},
$$

where $^{*}$ denotes the complex conjugate. $H_{p}(\mathbf{x}, \mathbf{k}, \omega), H_{v}(\mathbf{x}, \mathbf{k}, \omega)$ are sensitivity functions for the radiated pressure and the normal fluid particle velocity on the surface of structure, respectively. The sensitivity functions in the spatial domain are related to the spectral sensitivity functions in the wavenumber domain $\tilde{\mathbf{k}}$, denoted by $\tilde{H}_{p}(\tilde{\mathbf{k}}, \mathbf{k}, \omega)$ and $\tilde{H}_{v}(\tilde{\tilde{\mathbf{k}}}, \mathbf{k}, \omega)$, by inverse Fourier transform as follows

$$
\begin{aligned}
H_{p}(\mathbf{x}, \mathbf{k}, \omega) & =\frac{1}{4 \pi^{2}} \int_{\infty} \tilde{H}_{p}(\tilde{\mathbf{k}}, \mathbf{k}, \omega) e^{\mathrm{i} \tilde{\mathbf{k}} \mathbf{x}} \mathrm{d} \tilde{\mathbf{k}} \\
H_{v}^{*}(\mathbf{x}, \mathbf{k}, \omega) & =\frac{1}{4 \pi^{2}} \int_{\infty} \tilde{H}_{v}^{*}(\tilde{\mathbf{k}}, \mathbf{k}, \omega) e^{-\mathrm{i} \tilde{\mathbf{k}} \mathbf{x}} \mathrm{d} \tilde{\mathbf{k}} .
\end{aligned}
$$

Using Eqs. (1)-(4), the radiated acoustic power of a planar structure under stochastic excitation can be written as follows [26]

$$
\Pi^{\mathrm{rad}}(\omega)=\operatorname{Re}\left[\left(\frac{1}{4 \pi^{2}}\right)^{2} \int_{\infty} \int_{\infty} \tilde{H}_{p}(\tilde{\mathbf{k}}, \mathbf{k}, \omega) \tilde{H}_{v}^{*}(\tilde{\mathbf{k}}, \mathbf{k}, \omega) \phi_{p p}(\mathbf{k}, \omega) \mathrm{d} \mathbf{k} \mathrm{d} \tilde{\mathbf{k}}\right]
$$


where

$$
\tilde{k}_{z}(\tilde{\mathbf{k}})=\left\{\begin{array}{ll}
\sqrt{k_{a}^{2}-\tilde{k}_{x}^{2}-\tilde{k}_{y}^{2}}, & k_{a}^{2} \geq \tilde{k}_{x}^{2}+\tilde{k}_{y}^{2} \\
\mathrm{i} \sqrt{\tilde{k}_{x}^{2}+\tilde{k}_{y}^{2}-k_{a}^{2}}, & \text { otherwise }
\end{array}\right\}
$$

and $k_{a}$ is the acoustic wavenumber, $\rho_{a}$ is the fluid density, and $\tilde{\mathbf{k}}=\left(\tilde{k}_{x}, \tilde{k}_{y}\right)$. Substituting Eq. (6) in Eq. (5), the radiated acoustic power can be written either in terms of sound pressure or normal fluid particle velocity sensitivity functions as follows

$$
\begin{gathered}
\Pi_{p}^{\mathrm{rad}}(\omega)=\operatorname{Re}\left[\frac{1}{16 \pi^{4} \rho_{a} \omega} \int_{\infty} \int_{\infty} \tilde{k}_{z}^{*}(\tilde{\mathbf{k}})\left|\tilde{H}_{p}(\tilde{\mathbf{k}}, \mathbf{k}, \omega)\right|^{2} \phi_{p p}(\mathbf{k}, \omega) \mathrm{d} \tilde{\mathbf{k}} \mathrm{d} \mathbf{k}\right] \\
\Pi_{v}^{\mathrm{rad}}(\omega)=\operatorname{Re}\left[\frac{\rho_{a} \omega}{16 \pi^{4}} \int_{\infty} \int_{\infty} \frac{1}{\tilde{k}_{z}(\tilde{\mathbf{k}})}\left|\tilde{H}_{v}(\tilde{\mathbf{k}}, \mathbf{k}, \omega)\right|^{2} \phi_{p p}(\mathbf{k}, \omega) \mathrm{d} \tilde{\mathbf{k}} \mathrm{d} \mathbf{k}\right]
\end{gathered}
$$

The subscripts ${ }_{p}$ and ${ }_{v}$ correspond to the formulations based on the pressure and velocity sensitivity functions, respectively. Considering that the $\phi_{p p}(\mathbf{k}, \omega)$ is always real, the only function which could make the integrand in Eqs. (8) and $(9)$ complex is $\tilde{k}_{z}(\tilde{\mathbf{k}})$. According to Eq. (7), $\tilde{k}_{z}(\tilde{\mathbf{k}})$ becomes purely imaginary when the wavenumbers are outside the acoustic circle defined by $\Omega_{a}=\left\{\tilde{\mathbf{k}} \in \mathbb{R}^{2},|\tilde{\mathbf{k}}| \leq k_{a}\right\}$. Therefore, only wavenumbers inside the acoustic circle contribute to the radiated acoustic power. Hence, Eqs. (8) and $(9)$ can be rewritten as

$$
\begin{aligned}
& \Pi_{p}^{\mathrm{rad}}(\omega)=\frac{1}{16 \pi^{4} \rho_{a} \omega} \int_{\infty} \int_{\tilde{\mathbf{k}} \in \Omega_{a}} \sqrt{k_{a}^{2}-\tilde{k}_{x}^{2}-\tilde{k}_{y}^{2}}\left|\tilde{H}_{p}\left(\tilde{k}_{x}, \tilde{k}_{y}, \mathbf{k}, \omega\right)\right|^{2} \phi_{p p}(\mathbf{k}, \omega) \mathrm{d} \tilde{\mathbf{k}} \mathrm{d} \mathbf{k},(10) \\
& \Pi_{v}^{\mathrm{rad}}(\omega)=\frac{\rho_{a} \omega}{16 \pi^{4}} \int_{\infty} \int_{\tilde{\mathbf{k}} \in \Omega_{a}} \frac{1}{\sqrt{k_{a}^{2}-\tilde{k}_{x}^{2}-\tilde{k}_{y}^{2}}}\left|\tilde{H}_{v}\left(\tilde{k}_{x}, \tilde{k}_{y}, \mathbf{k}, \omega\right)\right|^{2} \phi_{p p}(\mathbf{k}, \omega) \mathrm{d} \tilde{\mathbf{k}} \mathrm{d} \mathbf{k} \cdot(11)
\end{aligned}
$$




\section{Non-Negative Intensity}

In this section, an analytical formulation is presented for non-negative intensity (the active normal intensity) for planar structures under stochastic excitation to identify the areas of the vibrating structure that produce radiation to the far-field. The aim here is to develop a formula for $I^{N}(\mathbf{x}, \omega)$ which meets the two following conditions:

1. The NNI must be always non-negative. This will prevent acoustic shortcircuit in the adjacent areas on the surface of the structure.

2. When integrating the NNI over the infinite boundary surface, it must produce the total sound power.

To meet the first condition, similar to works by Marburg et al. [8] and Williams 9 the NNI can be defined as follows

$$
I^{N}(\mathbf{x}, \omega)=\frac{1}{4 \pi^{2}} \int_{\infty} \beta(\mathbf{x}, \mathbf{k}, \omega) \beta^{*}(\mathbf{x}, \mathbf{k}, \omega) \mathrm{d} \mathbf{k}=\frac{1}{4 \pi^{2}} \int_{\infty}|\beta(\mathbf{x}, \mathbf{k}, \omega)|^{2} \mathrm{~d} \mathbf{k},
$$

where $\beta(\mathbf{x}, \mathbf{k}, \omega)$ is a complex function which is not physically meaningful. It has been introduced in Eq. (12) to ensure that the NNI is always nonnegative by definition. This satisfies the necessary condition for defining the NNI. The second condition for the NNI states that the total radiated acoustic power must be obtained by integrating the NNI over the infinite boundary surface

$$
\Pi^{\mathrm{rad}}(\omega)=\int_{\infty} I^{N}(\mathbf{x}, \omega) \mathrm{d} \mathbf{x}
$$

Eq. (13) can be rewritten in terms of $\beta(\mathbf{x}, \mathbf{k}, \omega)$ as follows

$\Pi^{\mathrm{rad}}(\omega)=\frac{1}{4 \pi^{2}} \int_{\infty} \int_{\infty} \beta(\mathbf{x}, \mathbf{k}, \omega) \beta^{*}(\mathbf{x}, \mathbf{k}, \omega) \mathrm{d} \mathbf{k} \mathrm{d} \mathbf{x}=\frac{1}{4 \pi^{2}} \int_{\infty} \int_{\infty}|\beta(\mathbf{x}, \mathbf{k}, \omega)|^{2} \mathrm{~d} \mathbf{k} \mathrm{d} \mathbf{x} .(14)$

To meet the second condition, we propose two new formulae for $\beta(\mathbf{x}, \mathbf{k}, \omega)$, one in terms of pressure sensitivity function and the other one based on the sensitivity function of normal fluid particle velocity. Both formulae are 
dependent on the CSD function of the stochastic field. The two formulae are given by

$$
\begin{gathered}
\beta_{p}(\mathbf{x}, \mathbf{k}, \omega)=\frac{\sqrt{\phi_{p p}(\mathbf{k}, \omega)}}{4 \pi^{2} \sqrt{\rho_{a} \omega}} \int_{\tilde{\mathbf{k}} \in \Omega_{a}} \sqrt[4]{k_{a}^{2}-\tilde{k}_{x}^{2}-\tilde{k}_{y}^{2}} \tilde{H}_{p}\left(\tilde{k}_{x}, \tilde{k}_{y}, \mathbf{k}, \omega\right) e^{\mathrm{i} \tilde{\mathbf{k}} \mathbf{x}} \mathrm{d} \tilde{\mathbf{k}} . \\
\beta_{v}(\mathbf{x}, \mathbf{k}, \omega)=\frac{\sqrt{\rho_{a} \omega \phi_{p p}(\mathbf{k}, \omega)}}{4 \pi^{2}} \int_{\tilde{\mathbf{k}} \in \Omega_{a}} \frac{1}{\sqrt[4]{k_{a}^{2}-\tilde{k}_{x}^{2}-\tilde{k}_{y}^{2}}} \tilde{H}_{v}\left(\tilde{k}_{x}, \tilde{k}_{y}, \mathbf{k}, \omega\right) e^{\mathrm{i} \tilde{\mathbf{k}} \mathbf{x}} \mathrm{d} \tilde{\mathbf{k}} .
\end{gathered}
$$

As can be seen from Eqs. (15) and (16), the integral domain is confined within the acoustic circle $\left(\tilde{\mathbf{k}} \in \Omega_{a}\right)$ which means that $k_{a}^{2} \geq \tilde{k}_{x}^{2}+\tilde{k}_{y}^{2}$ and $\tilde{k}_{z}(\tilde{\mathbf{k}})$ is real. These wavenumbers are associated with supersonic waves as their trace speeds are faster than the speed of sound. Whilst for the wavenumbers outside the acoustic circle, $\tilde{k}_{z}(\tilde{\mathbf{k}})$ is purely imaginary and the corresponding waves are called subsonic waves since they travel at phase speeds less than the speed of sound. The purpose of defining NNI is to identify local surfaces on a structure that are contributing to the far-field radiated sound. It is the farfield sound pressure that is normally of interest in engineering applications because this is the quantity to which a potential observer is typically exposed. The NNI enables the design engineers to identify the locations of unwanted sources of sound on the structure that make the most significant contributions to the far field. Therefore, only contributions of supersonic waves are taken into account and the subsonic components, which are evanescent and do not propagate to the far-field, are excluded.

To prove that the two formulae given by Eqs. (15) and (16) result in the radiated sound power as that given by Eqs. (10) and (11), Eq. (14) should be evaluated using Eqs. (15) and (16). In what follows, the proof is given for $\beta_{v}(\mathbf{k}, \omega)$ and similar approach can be used to verify that $\beta_{p}(\mathbf{k}, \omega)$ also meets this condition. $\beta_{v}^{*}(\mathbf{k}, \omega)$ can be written as follows

$$
\beta_{v}^{*}(\mathbf{x}, \mathbf{k}, \omega)=\frac{\sqrt{\rho_{a} \omega \phi_{p p}(\mathbf{k}, \omega)}}{4 \pi^{2}} \int_{\tilde{\tilde{\mathbf{k}}} \in \Omega_{a}} \frac{1}{\sqrt[4]{k_{a}^{2}-\tilde{\tilde{k}}_{x}^{2}-\tilde{\tilde{k}}_{y}^{2}}} \tilde{H}_{v}^{*}\left(\tilde{\tilde{k}}_{x}, \tilde{\tilde{k}}_{y}, \mathbf{k}, \omega\right) e^{-\tilde{\tilde{\mathbf{k}} \mathbf{x}} \mathrm{d}} \mathrm{\tilde { \mathbf {k } }},(17)
$$

substituting Eqs. (16) and (17) into Eq. (14) 


$$
\begin{aligned}
& \Pi_{v}^{\mathrm{rad}}(\omega)=\frac{\rho_{a} \omega}{16 \pi^{4}} \int_{\infty} \int_{\tilde{\tilde{\mathbf{k}} \in \Omega_{a}}} \int_{\tilde{\mathbf{k}} \in \Omega_{a}} \frac{\tilde{H}_{v}\left(\tilde{k}_{x}, \tilde{k}_{y}, \mathbf{k}, \omega\right)}{\sqrt[4]{k_{a}^{2}-\tilde{k}_{x}^{2}-\tilde{k}_{y}^{2}}} \frac{\tilde{H}_{v}^{*}\left(\tilde{\tilde{k}}_{x}, \tilde{\tilde{k}}_{y}, \mathbf{k}, \omega\right)}{\sqrt[4]{k_{a}^{2}-\tilde{\tilde{k}}_{x}^{2}-\tilde{\tilde{k}}_{y}^{2}}} \mathrm{~d} \tilde{\mathbf{k}} \\
& \left(\frac{1}{4 \pi^{2}} \int_{\infty} e^{\mathrm{i}(\tilde{\mathbf{k}}-\tilde{\tilde{\mathbf{k}}}) \mathbf{x}} \mathrm{d} \mathbf{x}\right) \mathrm{d} \tilde{\tilde{\mathbf{k}}} \phi_{p p}(\mathbf{k}, \omega) \mathrm{d} \mathbf{k}
\end{aligned}
$$

using the integral in the parenthesis in Eq. (18) corresponds to the Dirac delta function which is given by [27]

$$
\frac{1}{4 \pi^{2}} \int_{\infty} e^{\mathrm{i}(\tilde{\mathbf{k}}-\tilde{\mathbf{k}}) \mathbf{x}} \mathrm{d} \mathbf{x}=\delta(\tilde{\mathbf{k}}-\tilde{\tilde{\mathbf{k}}})
$$

using this definition, Eq. (18) can be simplified to

$$
\Pi_{v}^{\mathrm{rad}}(\omega)=\frac{\rho_{a} \omega}{16 \pi^{4}} \int_{\infty} \int_{\tilde{\mathbf{k}} \in \Omega_{a}} \frac{1}{\sqrt{k_{a}^{2}-\tilde{k}_{x}^{2}-\tilde{k}_{y}^{2}}}\left|\tilde{H}_{v}\left(\tilde{k}_{x}, \tilde{k}_{y}, \mathbf{k}, \omega\right)\right|^{2} \phi_{p p}(\mathbf{k}, \omega) \mathrm{d} \tilde{\mathbf{k}} \mathrm{d} \mathbf{k} \cdot(20)
$$

This equation is exactly the same as Eq. (11). The NNI formulae can be obtained by substituting Eqs. (15) and (16) into Eq. (12)

$I_{p}^{N}(\mathbf{x}, \omega)=\left.\frac{1}{\left(4 \pi^{2}\right)^{3} \rho_{a} \omega} \int_{\infty} \int_{\tilde{\mathbf{k}} \in \Omega_{a}} \sqrt[4]{k_{a}^{2}-\tilde{k}_{x}^{2}-\tilde{k}_{y}^{2}} \tilde{H}_{p}\left(\tilde{k}_{x}, \tilde{k}_{y}, \mathbf{k}, \omega\right) e^{\mathrm{i} \tilde{\mathbf{k}} \mathbf{x}} \mathrm{d} \tilde{\mathbf{k}}\right|^{2}\left|\phi_{p p}(\mathbf{k}, \omega)\right| \mathrm{d} \mathbf{k}$,

$I_{v}^{N}(\mathbf{x}, \omega)=\left.\frac{\rho_{a} \omega}{\left(4 \pi^{2}\right)^{3}} \int_{\infty} \int_{\tilde{\mathbf{k}} \in \Omega_{a}} \frac{1}{\sqrt[4]{k_{a}^{2}-\tilde{k}_{x}^{2}-\tilde{k}_{y}^{2}}} \tilde{H}_{v}\left(\tilde{k}_{x}, \tilde{k}_{y}, \mathbf{k}, \omega\right) e^{\mathrm{i} \tilde{\mathbf{k}} \mathbf{x}} \mathrm{d} \tilde{\mathbf{k}}\right|^{2}\left|\phi_{p p}(\mathbf{k}, \omega)\right| \mathrm{d} \mathbf{k} .(22)$

Due to the magnitude operation, these formulae are guaranteed to yield nonnegative results.

In Eq. (22), the term in the denominator tends to zero for the wavenumbers on the acoustic circle. Generally, singular integrals can be numerically 
197

$$
\begin{array}{r}
I_{p}^{N}(\mathbf{x}, \omega)=\frac{1}{\left(4 \pi^{2}\right)^{3} \rho_{a} \omega} \sum_{\mathbf{k} \in \Omega_{t}}\left|\left(\sum_{\tilde{\mathbf{k}} \in \Omega_{a}} \sqrt[4]{k_{a}^{2}-\tilde{k}_{x}^{2}-\tilde{k}_{y}^{2}} \tilde{H}_{p}\left(\tilde{k}_{x}, \tilde{k}_{y}, \mathbf{k}, \omega\right) e^{i \tilde{\mathbf{k}} \mathbf{x}} \delta \tilde{\mathbf{k}}\right)\right|^{2} \\
\left|\phi_{p p}(\mathbf{k}, \omega)\right| \delta \mathbf{k} .
\end{array}
$$

Finally, the change of variable, $\tilde{k}_{r}=k_{a} \sin \gamma$ analytically removes the singularity from the integral. As such, Eq. (24) can be expressed by

$$
\begin{array}{r}
I_{v}^{N}(\mathbf{x}, \omega)=\frac{\rho_{a} \omega k_{a}^{3}}{\left(4 \pi^{2}\right)^{3}} \int_{\infty} \mid\left(\int_{\theta=0}^{\theta=2 \pi} \int_{\gamma=0}^{\gamma=\frac{\pi}{2}} \sin \gamma \sqrt{\cos \gamma} e^{\mathrm{i} k_{a} \sin \gamma(x \cos \theta+y \sin \theta)}\right. \\
\left.\tilde{H}_{v}\left(k_{a} \sin \gamma \cos \theta, k_{a} \sin \gamma \sin \theta, \mathbf{k}, \omega\right) \mathrm{d} \gamma \mathrm{d} \theta\right)\left.\right|^{2}\left|\phi_{p p}(\mathbf{k}, \omega)\right| \mathrm{d} \mathbf{k},
\end{array}
$$

the rectangular method for the numerical integration in Eqs. (25) and (21), the NNI becomes

$$
\begin{array}{r}
I_{v}^{N}(\mathbf{x}, \omega)=\frac{\rho_{a} \omega k_{a}^{3}}{\left(4 \pi^{2}\right)^{3}} \sum_{\mathbf{k} \in \Omega_{t}} \mid\left(\sum_{\theta \in[0,2 \pi]} \sum_{\gamma \in\left[0, \frac{\pi}{2}\right]} \sin \gamma \sqrt{\cos \gamma} e^{\mathrm{i} k_{a} \sin \gamma(x \cos \theta+y \sin \theta)}\right. \\
\left.\tilde{H}_{v}\left(k_{a} \sin \gamma \cos \theta, k_{a} \sin \gamma \sin \theta, \mathbf{k}, \omega\right) \delta \gamma \delta \theta\right)\left.\right|^{2}\left|\phi_{p p}(\mathbf{k}, \omega)\right| \delta \mathbf{k},
\end{array}
$$

$\Omega_{t}$ is a truncated wavenumber domain and $\delta \gamma, \delta \theta, \delta \mathbf{k}$ and $\delta \tilde{\mathbf{k}}$ are the increments in the numerical integration. For the ADF excitation, since the 


\section{Application to Rectangular Baffled Panels}

A rectangular baffled panel excited by a stochastic pressure field is shown in Figure 1. The spatial average of the auto spectrum density (ASD) of the panel velocity is given by 30 32

$$
S_{v v}(\mathbf{x}, \omega)=\frac{1}{4 \pi^{2}} \int_{\infty}\left|H_{v_{s}}(\mathbf{x}, \mathbf{k}, \omega)\right|^{2} \phi_{p p}(\mathbf{k}, \omega) \mathrm{d} \mathbf{k}
$$

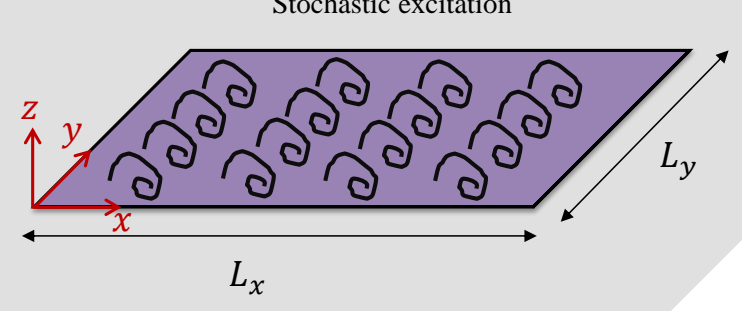

Figure 1: A baffled panel under stochastic excitation.

where $H_{v_{s}}(\mathbf{x}, \mathbf{k}, \omega)$ is the sensitivity function of the panel velocity excited by a unit wall plane wave. The spatial average of the ASD of the panel velocity 
217

is given by

$$
\left\langle V^{2}\right\rangle=\frac{1}{A} \int_{A} S_{v v}(\mathbf{x}, \omega) \mathrm{d} A
$$

$A=L_{x} L_{y}$ is the panel surface area and $L_{x}, L_{y}$ are the panel length and width in the $x$ and $y$ directions, respectively. Eqs. (28) and (29) can be evaluated using rectangular method as described in Ref [32]

The ASD of the radiated pressure from the panel excited by the stochastic field is also given by

$$
S_{p p}(\mathbf{x}, \omega)=\frac{1}{4 \pi^{2}} \int_{\infty}\left|H_{p}(\mathbf{x}, \mathbf{k}, \omega)\right|^{2} \phi_{p p}(\mathbf{k}, \omega) \mathrm{d} \mathbf{k},
$$

assuming that CSD of the stochastic field is known, it can be seen from the equations in Sections 2-4 that to evaluate $\Pi^{\mathrm{rad}}, I_{\mathrm{act}}, I^{N}, S_{v v}$ and $S_{p p}$, the sensitivity functions of panel velocity, normal fluid particle velocity and radiated pressure have to be known. In what follows, determination of these sensitivity functions are discussed.

\subsection{Determination of the Sensitivity Functions}

For a simply supported rectangular panel excited by a unit wall plane wave, the sensitivity function $H_{v_{s}}(\mathbf{x}, \mathbf{k}, \omega)$ corresponding to the velocity at point $\mathbf{x}$ is given by 32

$$
H_{v_{s}}(\mathbf{x}, \mathbf{k}, \omega)=\mathrm{i} \omega \sum_{m=1}^{M} \sum_{n=1}^{N} \frac{\psi_{m n}(\mathbf{k}) \varphi_{m n}(\mathbf{x})}{\Omega\left(\omega_{m n}^{2}-\omega^{2}+\mathrm{i} \eta \omega \omega_{m n}\right)},
$$

$\Omega=\rho_{s} h L_{x} L_{y} / 4$ is the modal mass. The modal frequencies are given by

$$
\omega_{m n}=\sqrt{\frac{D}{\rho_{s} h}}\left(\left(\frac{m \pi}{L_{x}}\right)^{2}+\left(\frac{n \pi}{L_{y}}\right)^{2}\right),
$$

where $D=E h^{3} /\left(12\left(1-\nu^{2}\right)\right)$ is the flexural rigidity, $E$ is the Young's modulus and $\nu$ is Poisson's ratio. The modal forces $\psi_{m n}$ are calculated by integration over the panel surface as follows

$$
\psi_{m n}(\mathbf{k})=\int_{A} \varphi_{m n}(\mathbf{x}) e^{-\mathrm{i}\left(k_{x} x+k_{y} y\right)} \mathrm{dA}=I_{m}^{x}\left(k_{x}\right) I_{n}^{y}\left(k_{y}\right),
$$


where $\varphi_{m n}(\mathbf{x})$ are the panel mode shapes given by

$$
\varphi_{m n}(\mathbf{x})=\sin \left(\frac{m \pi x}{L_{x}}\right) \sin \left(\frac{n \pi y}{L_{y}}\right)
$$

and

$$
\left\{I_{s}^{r}\left(k_{r}\right) \mid(r, s)=(x, m) \vee(y, n)\right\}=\left\{\begin{array}{ll}
\left(\frac{s \pi}{L_{r}}\right) \frac{(-1)^{s} e^{-\mathrm{i}\left(k_{r} L_{r}\right)}-1}{k_{r}^{2}-\left(\frac{s \pi}{L_{r}}\right)^{2},} & k_{r} \neq \frac{s \pi}{L_{r}} \\
\frac{1}{2} \mathrm{i} L_{r}, & \text { otherwise }
\end{array}\right\}
$$

At the interface between the panel and the acoustic domain, the structural velocity $v_{s}$ is equal to fluid particle velocity $v$ in the normal direction, that is $H_{v}(\mathbf{x}, \mathbf{k}, \omega)=H_{v_{s}}(\mathbf{x}, \mathbf{k}, \omega)$. As such, the spectral sensitivity function of normal fluid particle velocity $\tilde{H}_{v}(\tilde{\mathbf{k}}, \mathbf{k}, \omega)$ can be obtained analytically using a Fourier transform as follows

$$
\tilde{H}_{v}(\tilde{\mathbf{k}}, \mathbf{k}, \omega)=\int_{\infty} H_{v}(\mathbf{x}, \mathbf{k}, \omega) e^{-\mathrm{i} \tilde{\mathbf{k}} \mathbf{x}} \mathrm{d} \mathbf{x}=\sum_{m=1}^{M} \sum_{n=1}^{N} a_{m n}(\tilde{\mathbf{k}}, \omega) \psi_{m n}(\mathbf{k}),
$$

where

$$
a_{m n}(\tilde{\mathbf{k}}, \omega)=\mathrm{i} \omega \frac{\psi_{m n}(\tilde{\mathbf{k}})}{\Omega\left(\omega_{m n}^{2}-\omega^{2}+\mathrm{i} \eta \omega \omega_{m n}\right)},
$$

and $\psi_{m n}$ and $I_{s}^{r}$ are given by Eqs. (33)-(35).

Since $\tilde{H}_{p}(\tilde{\mathbf{k}}, \mathbf{k}, \omega)$ is related to $\tilde{H}_{v}(\mathbf{k}, \mathbf{k}, \omega)$ by Eq. $(6)$, to obtain $H_{p}(\mathbf{x}, \mathbf{k}, \omega)$, one can compute the inverse Fourier transform of Eq. (6). However, in order to avoid an additional inverse Fourier transform we used an alternative approach based on the Lyamshev reciprocity principle [33, 34]. Figure 2 illustrates the Lyamshev reciprocity principle for a baffled panel.

According to Lyamshev reciprocity principle, the ratio of the pressure at point $\mathbf{x}$ over the applied normal force at point $\mathbf{x}^{\prime}$ is equal to the ratio of the normal velocity of the panel at point $\mathbf{x}^{\prime}$ over the volume velocity $Q_{v}$ of a monopole source placed at point $\mathbf{x}$, that is,

$$
H_{p / F}\left(\mathbf{x}, \mathbf{x}^{\prime}, \omega\right)=H_{v / Q_{v}}\left(\mathbf{x}^{\prime}, \mathbf{x}, \omega\right),
$$




$$
p(x) \circ \quad Q_{v}(x) \times
$$
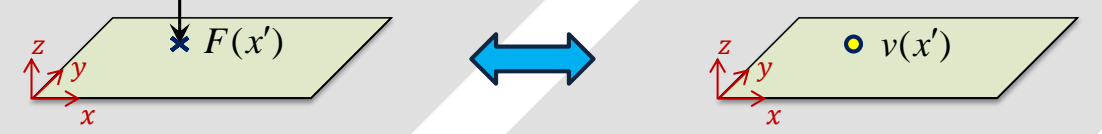

Figure 2: Illustration of the Lyamshev reciprocity principle for a baffled panel.

where

$$
H_{v / Q_{v}}\left(\mathbf{x}^{\prime}, \mathbf{x}, \omega\right)=\mathrm{i} \omega \sum_{m=1}^{M} \sum_{n=1}^{N} \frac{F_{m n}(\mathbf{x}) \varphi_{m n}\left(\mathbf{x}^{\prime}\right)}{\Omega\left(\omega_{m n}^{2}-\omega^{2}+\mathrm{i} \eta \omega \omega_{m n}\right)}
$$

and

$$
F_{m n}(\mathbf{x})=\int_{A} p\left(\mathbf{x}, \mathbf{x}^{\prime}, \omega\right) \varphi_{m n}\left(\mathbf{x}^{\prime}\right) \mathrm{d} \mathbf{x}^{\prime}
$$

where $p\left(\mathbf{x}, \mathbf{x}^{\prime}, \omega\right)$ is the acoustic pressure generated by a monopole source and is given by

$$
p\left(\mathbf{x}, \mathbf{x}^{\prime}, \omega\right)=\frac{\mathrm{i} \rho_{a} \omega Q_{v}}{2 \pi \mathbf{r}} e^{-\mathrm{i} k_{a} \mathbf{r}}, \quad \mathbf{r}=\left|\mathbf{x}-\mathbf{x}^{\prime}\right| .
$$

The sensitivity function of the radiated pressure is given by

$$
H_{p}(\mathbf{x}, \mathbf{k}, \omega)=\int_{\infty} H_{p / F}\left(\mathbf{x}, \mathbf{x}^{\prime}, \omega\right) e^{-\mathrm{ikx^{ \prime }}} \mathrm{d} \mathbf{x}^{\prime}
$$

substituting Eqs. (38)-(39) into Eq. (42), the sensitivity function $H_{p}(\mathbf{x}, \mathbf{k}, \omega)$ can be written as follows

$$
H_{p}(\mathbf{x}, \mathbf{k}, \omega)=\sum_{m=1}^{M} \sum_{n=1}^{N} a_{m n}(\mathbf{k}, \omega) F_{m n}(\mathbf{x}),
$$

where $F_{m n}(\mathbf{x})$ is given by Eq. (40) and can be numerically computed using rectangular method. 
Table 1. Dimensions and material properties of the panel

\begin{tabular}{lc}
\hline Parameter & Value \\
\hline Young's modulus, $E(\mathrm{GPa})$ & 70 \\
Poisson's ratio, $\nu$ & 0.3 \\
Mass density, $\rho_{s}\left(\mathrm{~kg} / \mathrm{m}^{3}\right)$ & 2700 \\
Length, $L_{x}(\mathrm{~mm})$ & 480 \\
Width, $L_{y}(\mathrm{~mm})$ & 420 \\
Thickness, $h_{s}(\mathrm{~mm})$ & 3.17 \\
Damping loss factor, $\eta$ & 0.005 \\
\hline
\end{tabular}

\section{Results and Discussion}

A rectangular baffled panel with simply-supported boundary conditions is considered. The dimensions and material properties of the panel are listed in Table 1. The fluid density and kinematic viscosity were set to $1.225 \mathrm{~kg} / \mathrm{m}^{3}$ and $1.511 \times 10^{-5} \mathrm{~m}^{2} / \mathrm{s}$, respectively.

\subsection{Modeling TBL and ADF Excitations}

The surface contributions of the panel to the radiated sound power under two different stochastic excitations, namely TBL and ADF are examined. The CSD of the stochastic field can be expressed in terms of the ASD function $\Psi_{p p}(\omega)$ and the normalized CSD function of the stochastic field $\tilde{\phi}_{p p}(\mathbf{k}, \omega)$ as follows [35; 36]

$$
\phi_{p p}(\mathbf{k}, \omega)=\Psi_{p p}(\omega) \tilde{\phi}_{p p}(\mathbf{k}, \omega) .
$$

Eq. (44) can be used to evaluate the CSD of both the ADF and TBL excitations. A unity ASD is assumed for both excitations. The normalized CSD functions given in Appendix A were also used to evaluate the TBL and ADF excitations, respectively. For TBL excitation, it is assumed that the TBL is stationary, homogeneous and fully developed over the panel surface. Moreover, it is assumed the vibration of the panel does not alter the wall pressure field (WPF). The Mellen model described in Appendix A was used to evaluate the CSD function of the WPF [37]. The TBL parameters were estimated based on theoretical formula for a flat panel from literature and are given in Table 2 [32]. The convective velocity $U_{c}$ was approximated as follows [32; 38]

$$
U_{c} \cong U_{\infty}\left(0.59+0.3 e^{-0.89 \delta^{*} \omega / U_{\infty}}\right),
$$


Table 2. TBL parameters at a flow speed of $40 \mathrm{~m} / \mathrm{s}$

\begin{tabular}{lc}
\hline Parameter & Value \\
\hline TBL thickness $\delta(\mathrm{m})$ & 0.0349 \\
TBL displacement thickness $\delta^{*}(\mathrm{~m})$ & 0.0044 \\
Wall shear stress $\tau(\mathrm{Pa})$ & 2.5228 \\
\hline
\end{tabular}

where $U_{\infty}$ is the free flow velocity and $\delta^{*}$ is boundary layer displacement thickness.

\subsection{Determination of Cut-off Wavenumbers and Wavenumber Resolutions}

It has previously been reported when a panel is excited by a TBL, the effect of convected ridge can be neglected for frequencies well above the aerodynamic frequency [26; 32]. Therefore, to predict the vibroacoustic response of the panel the cut-off wavenumber can be defined based on the flexural wavenumber. This is due to the filtering effect of the structure. In this study, it was confirmed that the same criterion can be used to evaluate the NNI. One can plot the forcing function and sensitivity function to illustrate the filtering effect. To do this, Eq. (22) can be further written in a compact form as follows

$$
I_{v}^{N}(\mathbf{x}, \omega)=\left(\frac{1}{4 \pi^{2}}\right) \int_{\infty}\left|\tilde{H}_{N}(\mathbf{x}, \mathbf{k}, \omega)\right|^{2} \phi_{p p}(\mathbf{k}, \omega) \mathrm{d} \mathbf{k},
$$

where $\tilde{H}_{N}(\mathbf{x}, \mathbf{k}, \omega)$ is the NNI sensitivity function given by

$$
\begin{array}{r}
\tilde{H}_{N}(\mathbf{x}, \mathbf{k}, \omega)=\frac{\sqrt{\rho_{a} \omega k_{a}^{3}}}{4 \pi^{2}}\left(\sum_{\theta \in[0,2 \pi]} \sum_{\gamma \in\left[0, \frac{\pi}{2}\right]} \sin \gamma \sqrt{\cos \gamma} e^{\mathrm{i} k_{a} \sin \gamma(x \cos \theta+y \sin \theta)}\right. \\
\left.\tilde{H}_{v}\left(k_{a} \sin \gamma \cos \theta, k_{a} \sin \gamma \sin \theta, \mathbf{k}, \omega\right) \delta \gamma \delta \theta\right)
\end{array}
$$

Figure 3(a) presents a map of the NNI sensitivity function at $(x, y)=$ $(0.4 \mathrm{~m}, 0.4 \mathrm{~m})$ and for $k_{y}=0$. The black dashed lines correspond to the panel flexural wavenumbers. It can be seen that the sensitivity function reaches its maximum values at wavenumbers smaller than or close to the flexural wavenumbers. However, for the wavenumbers larger than the flexural wavenumbers the magnitude of the function is still considerable, particularly 
at resonance frequencies. Figure 3 (b) shows the TBL forcing function, corresponding to the CSD of the WPF. The convective wavenumbers are denoted by the dash-dotted line. Figure 3(c) presents the product of the sensitivity function and forcing function. It can be observed from Figure 3(c) that most of the wavenumbers larger than flexural wavenumber are filtered out. Therefore, only wavenumbers smaller than flexural wavenumbers contribute to the NNI. However, a small effect of the convective ridge on the product of the sensitivity function and forcing function can be observed around $150 \mathrm{~Hz}$ and $350 \mathrm{~Hz}$. Whilst this contribution is not significant, the effect of the convective ridge was taken into account here as the cut-off wavenumber was defined as twice the flexural wavenumber at the highest frequency of interest. In fact, a cut-off wavenumber of $k_{\text {cut-off }}=2 k_{p \text {,max }}$ was selected. Therefore, a wavenumber range of $\left[-2 k_{p, \max }, 2 k_{p, \max }\right]$ was used in both the streamwise and spanwise directions where $k_{p, \max }=\left(\omega_{\max } \sqrt{\rho_{s} h / D}\right)^{1 / 2}$ is the flexural wavenumber of the panel at the maximum frequency of interest denoted by $\omega_{\max }$. The wavenumber resolutions were set to $\delta k_{x}=\delta k_{y}=0.25(1 / \mathrm{m})$, and $\delta \gamma, \delta \theta$ were set to $\pi / 60$. These values were determined using a convergence study. It should also be pointed out that although the NNI sensitivity function was plotted at a certain point on the panel, the same filtering effect occurs for all the points on the panel and similar behavior could be observed if the maps were plotted at a different point. 


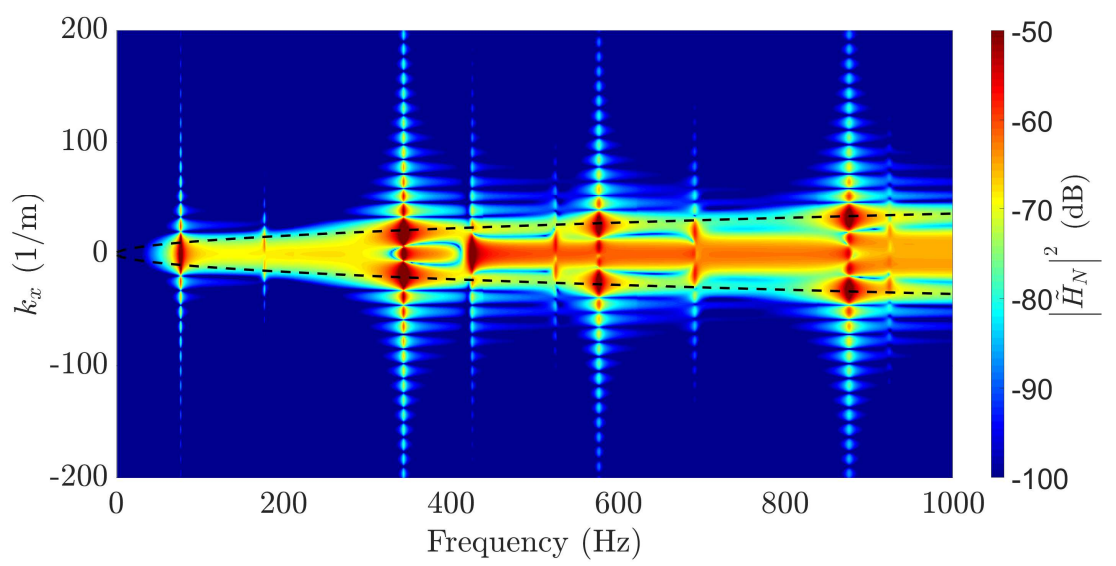

(a)

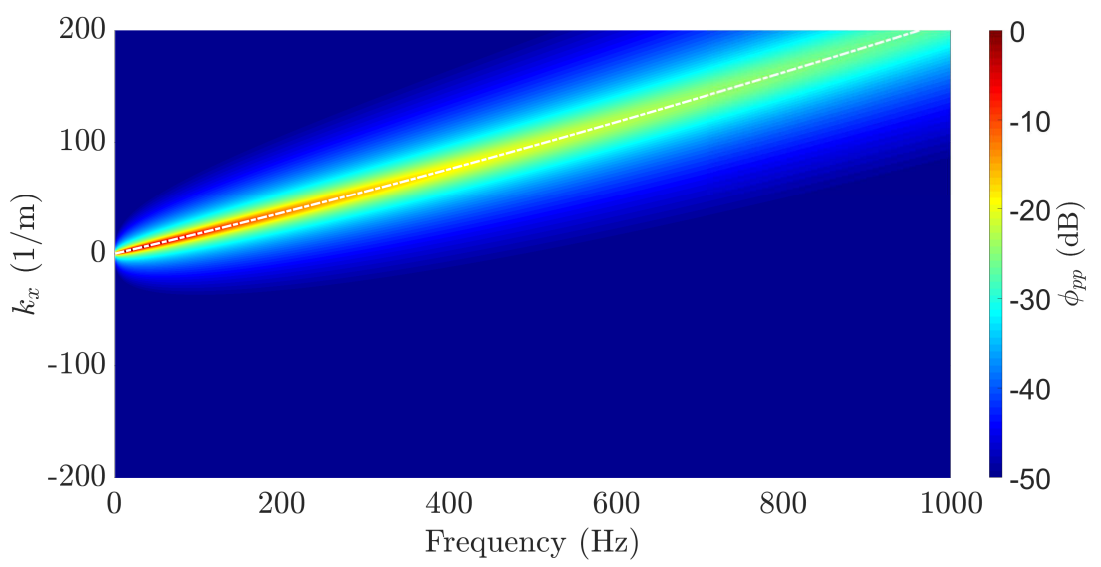

(b)

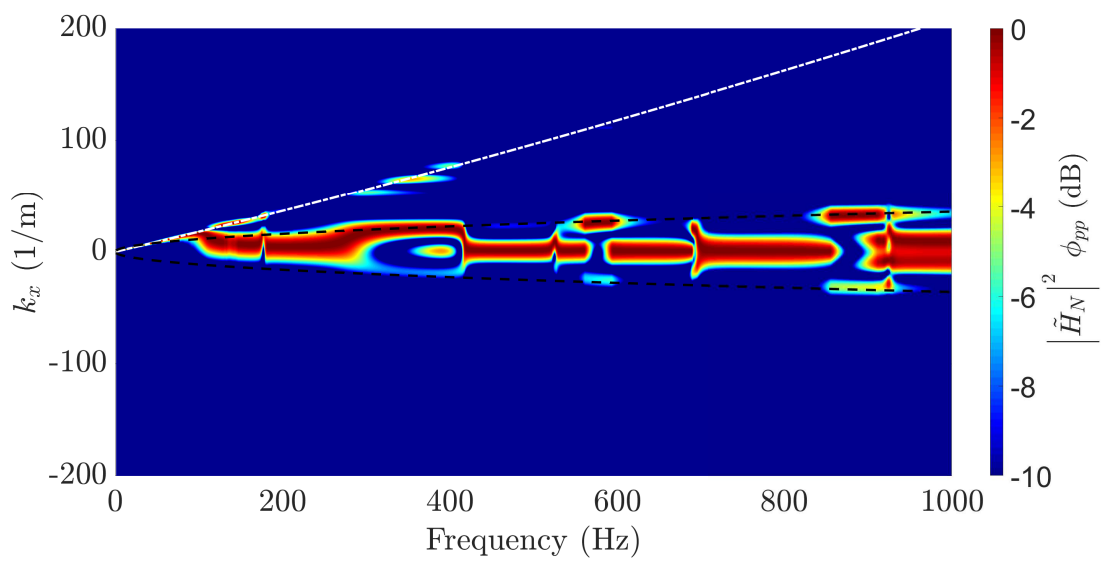

(c)

Figure 3: Maps of the (a) NNI sedstivity functions $\left|\tilde{H}_{N}(\mathbf{x}, \mathbf{k}, \omega)\right|^{2}$ (dB, ref. $\mathrm{Pa}^{-1} \mathrm{~m}^{3} \mathrm{~s}^{-2} \mathrm{rad}^{2}$ ), (b) CSD function of the wall pressure spectrum using the Mellen model $\phi_{p p}(\mathbf{k}, \omega)\left(\mathrm{dB}\right.$, ref. $\left.1 \mathrm{~Pa}^{2} \mathrm{~m}^{2} \mathrm{~s} \mathrm{rad}^{-2}\right)$, and (c) result obtained by the product of (a) and (b) normalized by the maximum value at each frequency $\left(\mathrm{dB}\right.$, ref. $\left.1 \mathrm{Wm}^{2}\right)$. The black dashed lines in (a) and (c) correspond to the panel flexural wavenumber; the white dashed-dot line in (b) and (c) corresponds to the convective wavenumber. 


\subsection{Vibroacoustic Response of the Panel}

Figures 4 and 5 respectively present the spatial average of the ASD of the panel velocity and the radiated sound power of the panel under the TBL and ADF excitations. The TBL excitation strongly excites the structure at the aerodynamic coincidence frequency, $f_{c}$, which occurs when the flexural wavenumber given by $k_{p}=\left(\omega \sqrt{\rho_{s} h / D}\right)^{1 / 2}$ is equal to the convective wavenumber $k_{c}=\omega / U_{c}$, that is, $f_{c}=U_{c}^{2} \sqrt{\rho_{s} h / D} /(2 \pi)$ [39]. For the parameters chosen here and at a flow speed of $40 \mathrm{~m} / \mathrm{s}, f_{c}=29 \mathrm{~Hz}$. It can be seen from both figures that except at very low frequencies the spectral levels of the velocity and the sound power of the panel under the ADF excitation are significantly higher than those for the panel excited by the TBL (a unity ASD of the stochastic field was assumed for both excitations). Further, the shape and trend of the panel velocity response under the TBL excitation is very similar to that under the ADF excitation. However, a different behavior for the radiated sound power can be observed in Figure 5. The radiated sound power between resonance frequencies for the ADF excitation is relatively flat whilst the sound power at those frequencies form a curved shape in the spectra for the TBL excitation.

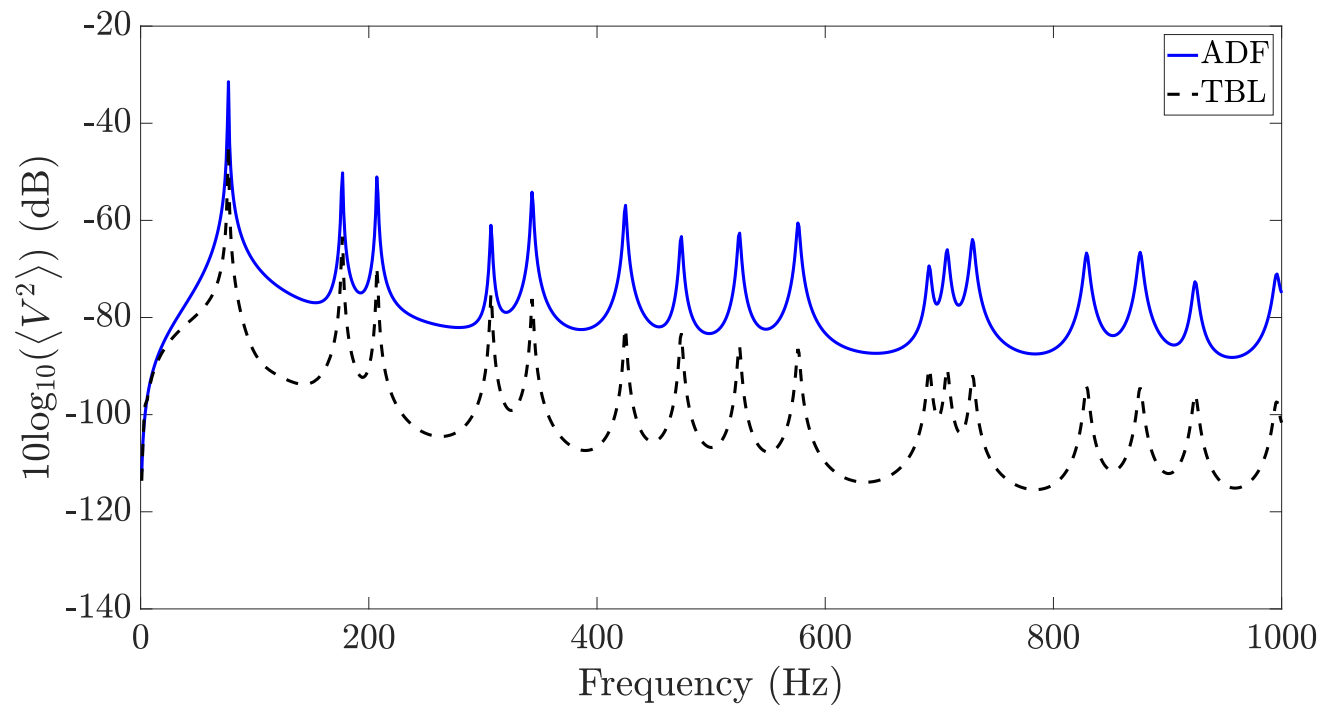

Figure 4: Predicted mean quadratic velocity spectra for the TBL and ADF excitations $\left(\mathrm{dB}\right.$ ref. $\left.1(\mathrm{~m} / \mathrm{s})^{2} / \mathrm{Hz}\right)$. 


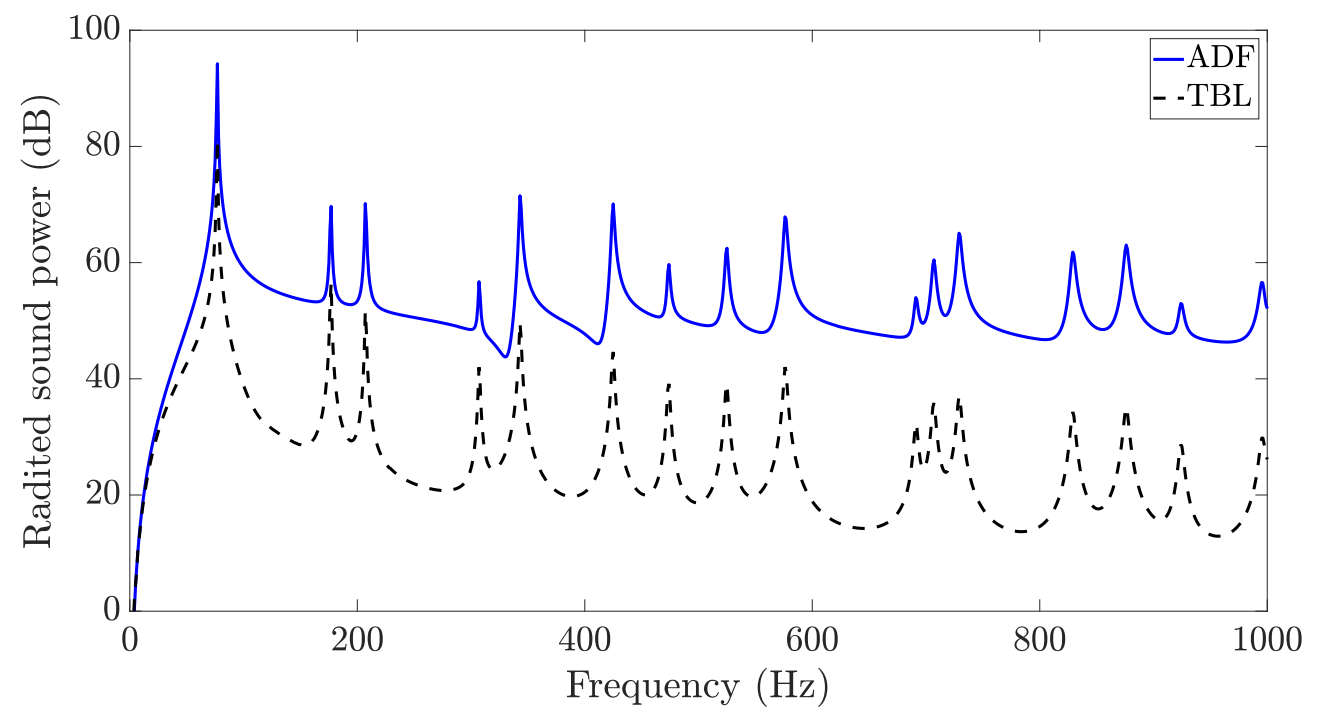

Figure 5: Predicted acoustic power of the panel under the TBL and ADF excitations (dB ref. $\left.1 \times 10^{-12}(\mathrm{~W})\right)$.

Figure 6 shows the radiation efficiency of the panel for both the ADF and TBL excitations. The radiation efficiency of a panel is given by [40]

$$
\sigma=\frac{\Pi_{\mathrm{rad}}}{A \rho_{a} c_{a}\left\langle V^{2}\right\rangle},
$$

vertical lines in Figure 6 indicate the resonance frequencies of the panel, the mode number for each resonance frequency has also been shown $((m, n)$ mode means an $m$ mode in the $x$-direction and an $n$ mode in the $y$-direction). It can be observed from Figure 6 that at very low frequencies the radiation efficiency of the panel is independent of the excitation force, and at higher frequencies the radiation efficiency of the panel under the ADF excitation is generally higher than that of the panel excited by the TBL, particularly at non-resonance frequency, the ADF excited panel efficiently radiates sound to the acoustic domain. At resonance frequency, the radiation frequency is almost the same for both excitations. 


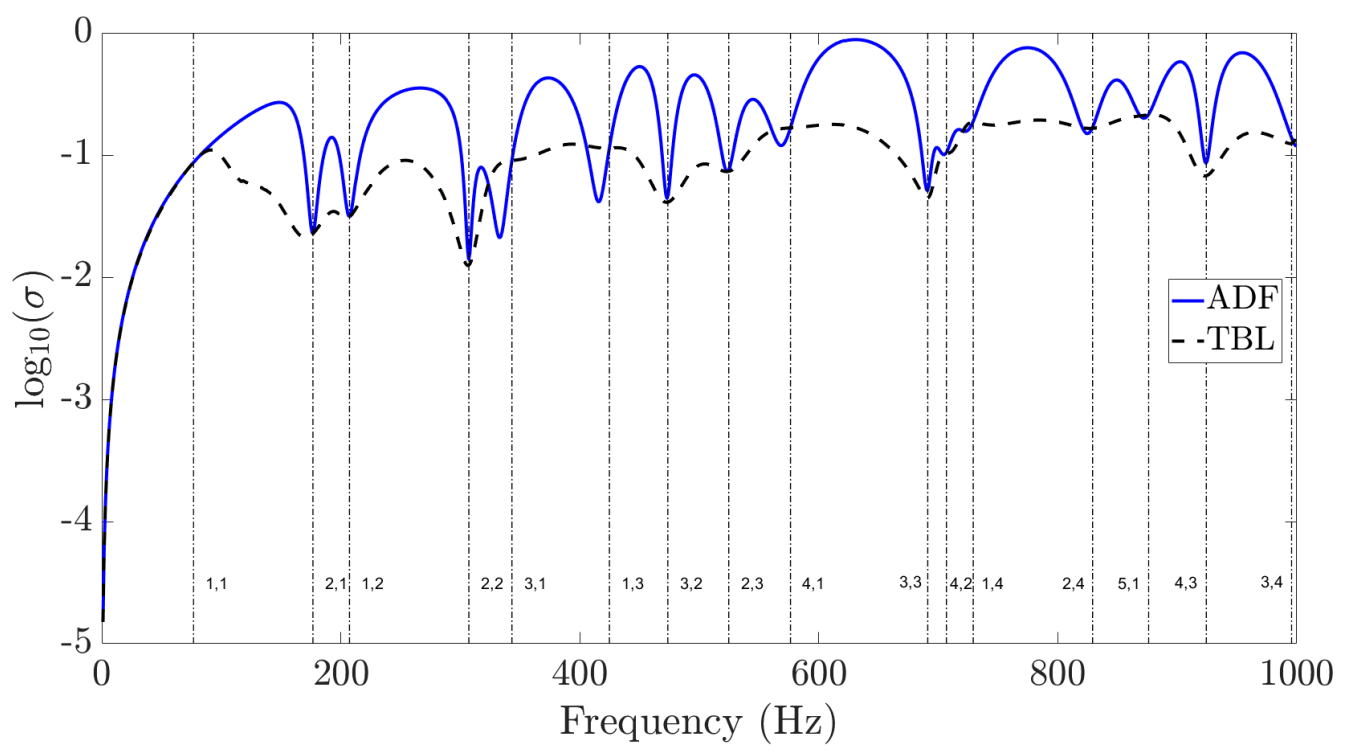

Figure 6: Radiation efficiency of the panel under the TBL and ADF excitations.

\subsection{The NNI Calculation}

To identify the surface contributions of the panel to the radiated sound power under the ADF and TBL excitations, the NNI has been computed at four discrete resonance frequencies of $177 \mathrm{~Hz}, 307 \mathrm{~Hz}, 691 \mathrm{~Hz}$ and $924 \mathrm{~Hz}$ as well as at two non-resonance frequencies of $630 \mathrm{~Hz}$ and $700 \mathrm{~Hz}$. The maps of $S_{v v}, S_{p p}, I_{\text {act }}$ and $I^{N}$ at the selected frequencies are presented in Figures 7 and 8 for the panel under the TBL and ADF excitations, respectively. It can be observed that regardless of excitation, at each frequency (particularly at the resonance frequencies) the map of $S_{v v}$ is very similar to that of $S_{p p}$. This is not surprising as $S_{p p}$ was evaluated on the surface of the panel, and the sensitivity functions of velocity and pressure have similar characteristic and are related to each other by Eq. (6). Figures 7 and 8 show that the active normal intensity $I_{\text {act }}$ of the panel excited by the ADF is higher than that under the TBL excitation, this is consistent with the sound power results presented in Figure 5. Further, it can be seen that the maps of $I_{\text {act }}$ for both excitations are very similar and the patterns at the resonance frequencies are highly dominated by the mode shapes.

For the TBL excitation, the NNI shows a distribution where mainly the edges and corners of the panel are significantly contributing to the radiated sound. This is consistent with the concept of edge and corner modes 
introduced by Maidanik [41]. For example, at $177 \mathrm{~Hz}$ the edge mode is contributing to the far-field sound power while at $307 \mathrm{~Hz}, 691 \mathrm{~Hz}, 700 \mathrm{~Hz}$ and $924 \mathrm{~Hz}$ the corner modes are the main contributor. At $630 \mathrm{~Hz}$, a large surface located between the center and two edges of the panel generates propagative waves to the far field. For the ADF excitation at $177 \mathrm{~Hz}$ and $924 \mathrm{~Hz}$ a similar NNI distribution to those of TBL excitation in Figure 7 can be observed. At these two resonance frequencies, regardless of excitation, only edge and corner modes are contributing to the radiated sound. Figures 7 and 8 show that the NNI distribution for the panel under the TBL excitation at $307 \mathrm{~Hz}$ and $700 \mathrm{~Hz}$ are mainly at the corners of the panel while for the ADF excitation the NNI is distributed along the diagonal of the panel with high intensity in the middle of the panel. Further, at $630 \mathrm{~Hz}$ the hot spots are formed as two separate vertical ellipses for the TBL excited panel while for the ADF excited panel the NNI was contained within a large horizontal ellipse. According to Figures 7 and 8 , in addition to the corner modes which effectively generate supersonic waves to the far field for both excitations at $691 \mathrm{~Hz}$, there is a hot spot in the middle of the panel for the ADF excitation which radiates energy to the far field. Results in Figures 7 and 8 revealed that the NNI distribution depends on the excitation type and frequency.

It should be noted that since normal fluid particle velocity is zero over the baffle (outside the panel surface), the active normal intensity is also zero everywhere on the baffle. Therefore, plotting $I_{\text {act }}$ over the panel surface shows the total intensity pattern, and the total radiated sound power can be evaluated by taking the integral of $I_{\text {act }}$ over the panel surface. However, the NNI is not necessarily zero on the baffle. To obtain the total sound power from the NNI, its entire distribution over the infinite boundary surface has to be considered as indicated by Eq. (13). Hence, the whole NNI distributions are plotted over a large boundary surface at $z=0$ for the selected frequencies as shown in Figures 9 and 10. The solid white lines in the maps indicate the rectangular panel under ADF/TBL excitations. Figures 9 and 10 show that the maxima of the NNI are usually located outside the panel surface, particularly at low frequencies as shown in Figure 9(a) and (b). The NNI distributions shown in Figures 7 and 8 are basically small parts of the whole distributions at most selected frequencies. The total NNI distribution in Figures 9 and 10 can be considered as an image of the excitation sources viewed by the acoustic domain. For instance, Figure 9 shows that at $177 \mathrm{~Hz}$ the size of each hot spot is around $1 \mathrm{~m}$ which corresponds to the half acoustic wavelength. Hence, the spatial resolution of the NNI is directly related to 


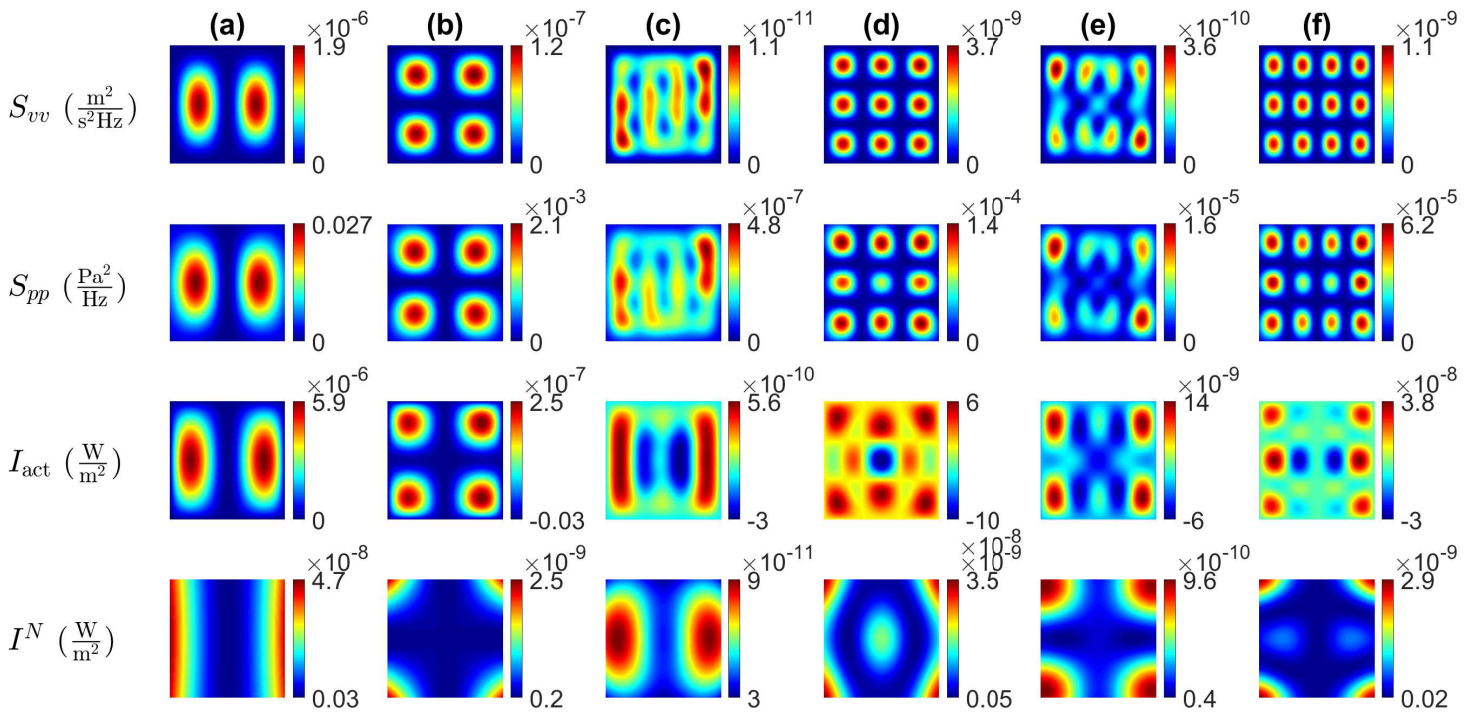

Figure 7: Maps of $S_{v v}, S_{p p}, I_{\text {act }}$ and $I^{N}$ for the panel under the TBL excitation at a flow velocity of $U_{\infty}=40 \mathrm{~m} / \mathrm{s}$ and at selected frequencies of (a) $177 \mathrm{~Hz}$, (b) $307 \mathrm{~Hz}$, (c) $630 \mathrm{~Hz}$, (d) $691 \mathrm{~Hz},(\mathrm{e}) 700 \mathrm{~Hz}$ and (f) $924 \mathrm{~Hz}$. 


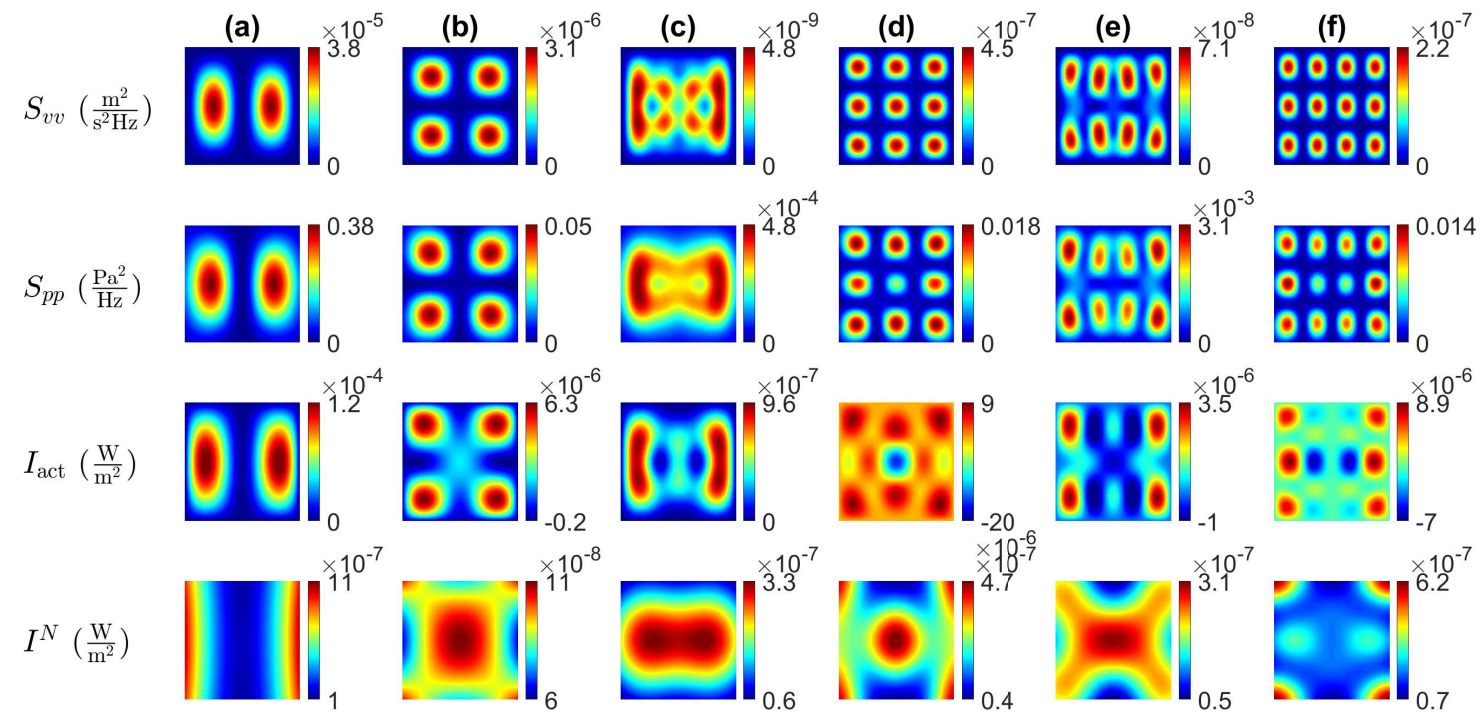

Figure 8: Maps of $S_{v v}, S_{p p}, I_{\text {act }}$ and $I^{N}$ for the panel under ADF excitation at a flow velocity of $U_{\infty}=40 \mathrm{~m} / \mathrm{s}$ and at selected frequencies of (a) $177 \mathrm{~Hz}$, (b) $307 \mathrm{~Hz}$, (c) $630 \mathrm{~Hz}$, (d) $691 \mathrm{~Hz}$, (e) $700 \mathrm{~Hz}$ and (f) $924 \mathrm{~Hz}$. 

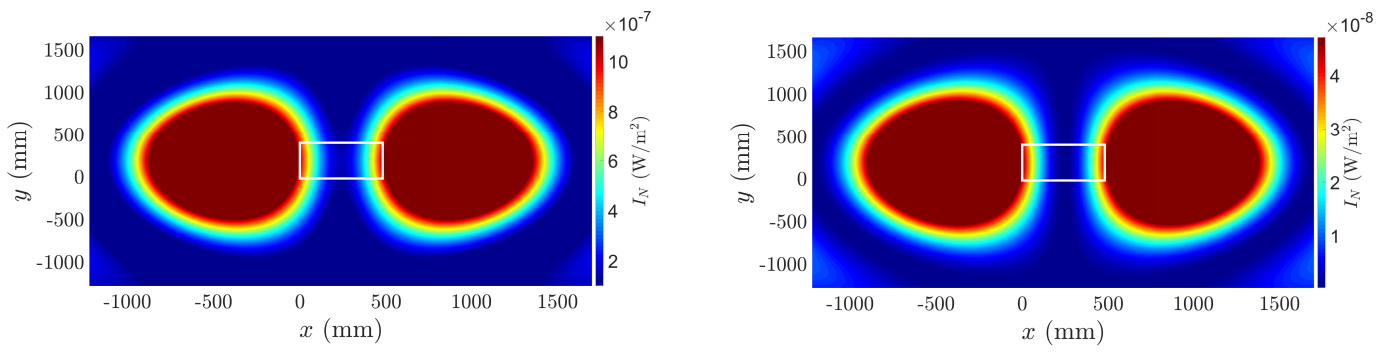

(a)
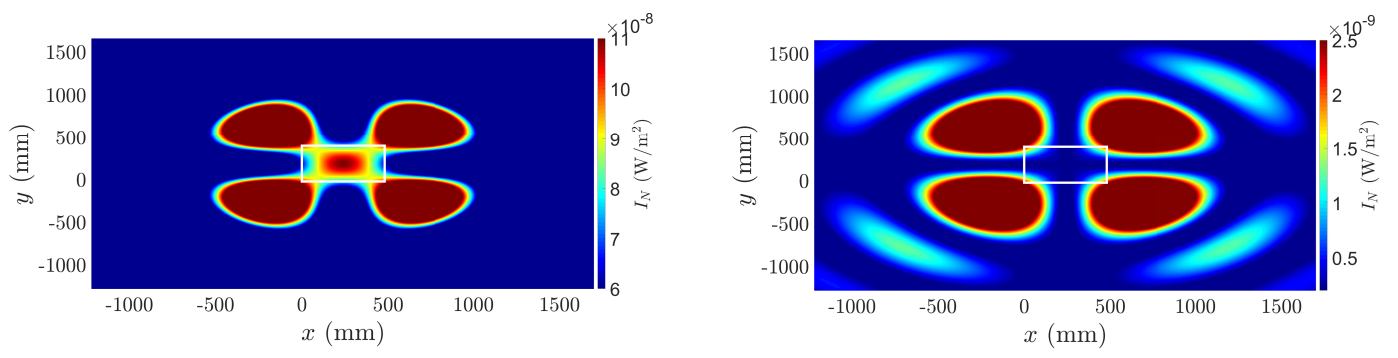

(b)
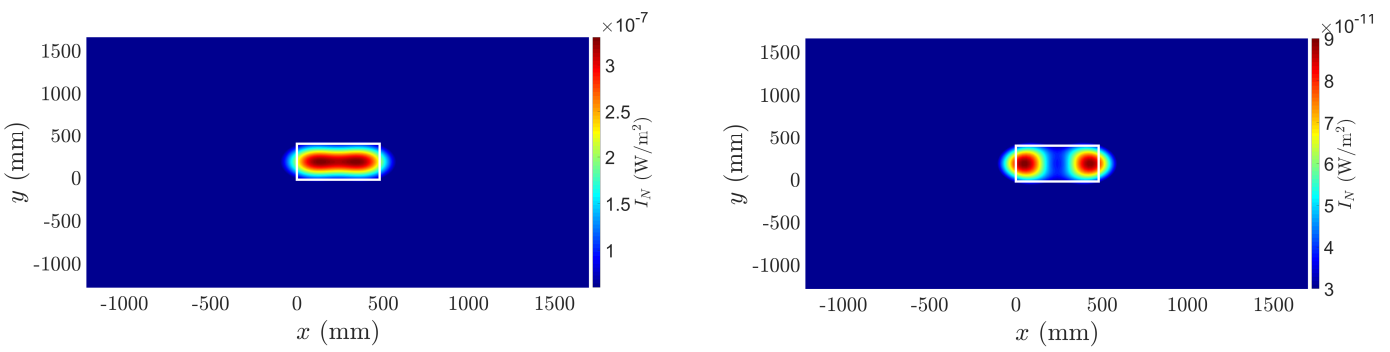

(c)

Figure 9: Comparison of the NNI between the panel under ADF excitation (left column) and under TBL excitation (right column) over a large surface at $z=0$ for selected frequencies of (a) $177 \mathrm{~Hz}$, (b) $307 \mathrm{~Hz}$, (c) $630 \mathrm{~Hz}$. 

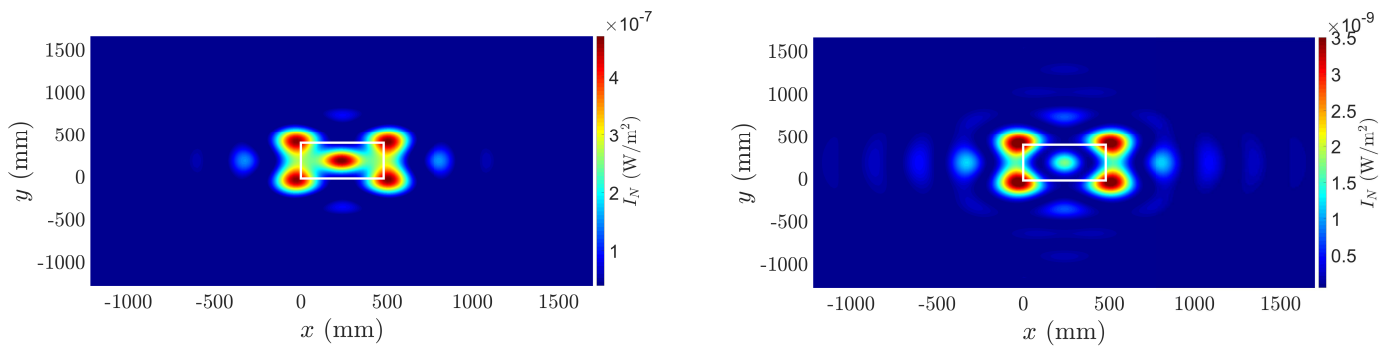

(a)
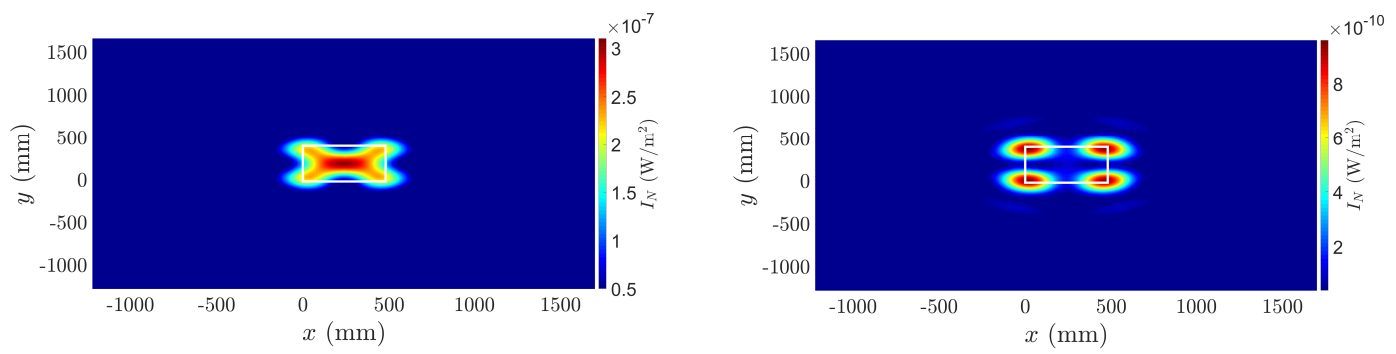

(b)
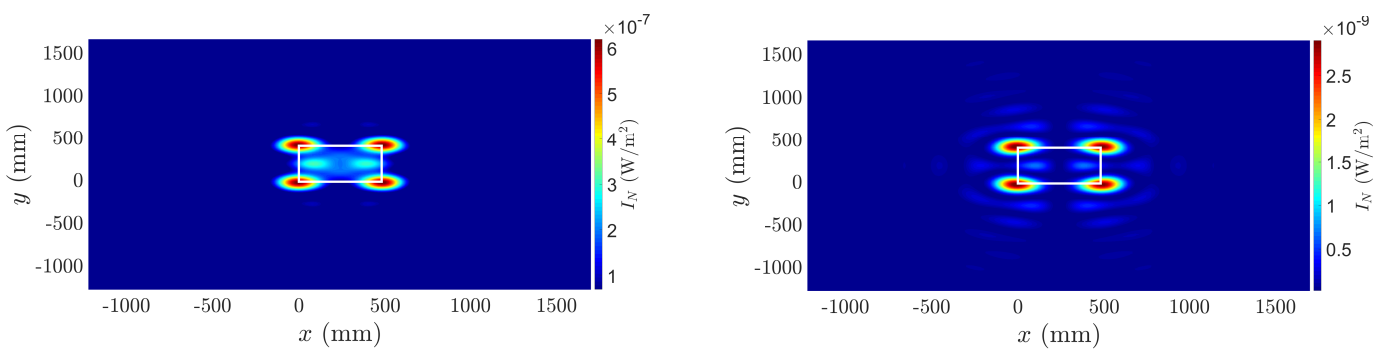

(c)

Figure 10: Comparison of the NNI between the panel under ADF excitation (left column) and under TBL excitation (right column) over a large surface at $z=0$ for selected frequencies of (a) $691 \mathrm{~Hz}$, (b) $700 \mathrm{~Hz}$ and (c) $924 \mathrm{~Hz}$. 


\section{Conclusions}

The non-negative intensity was analytically formulated in wavenumber domain for planar structures subject to random excitations. To calculate the NNI, the CSD of the stochastic field and either the sensitivity function of pressure or normal fluid particle velocity were required. The proposed formulation can be used for both infinite planar structure and finite plate in an infinite baffle. The NNI was used to quantify the regions on a simply supported baffled panel excited by the TBL and ADF which radiate energy to the far field. Comparing maps of the ASD of the pressure and panel velocity, and active intensity with those of the NNI at different frequencies revealed that the NNI is a powerful tool to identify hot spots on the panel surface which contribute to the sound power. It was also found that the NNI distribution is dependent on the excitation type as well as on the frequency of excitation. It was shown that the more the NNI distribution is concentrated within the panel surface, the higher the radiation efficiency becomes. In other word, high radiation efficiency can be achieved if the most area of the panel contributes to the radiated sound power, and this can be identified using the NNI.

\section{Acknowledgments}

This research was supported by the Australian Government through the Australian Research Council's Discovery Early Career Project funding scheme (project DE190101412).

\section{Appendix A: The normalized CSD function of TBL and ADF ex- citations}

\section{The Mellen model}

The Mellen normalized wavenumber-frequency model is given by [37]

$$
\tilde{\phi}_{p p}^{\mathrm{TBL}}\left(k_{x}, k_{y}, \omega\right)=\frac{2 \pi\left(\alpha_{x} \alpha_{y}\right)^{2} k_{\mathrm{c}}}{\left(\left(\alpha_{x} \alpha_{y} k_{\mathrm{c}}\right)^{2}+\left(\alpha_{x} k_{y}\right)^{2}+\alpha_{y}^{2}\left(k_{x}-k_{\mathrm{c}}\right)^{2}\right)^{3 / 2}}
$$

where $k_{c}=\omega / U_{c}, \alpha_{x}=0.1$ and $\alpha_{y}=0.77$ 
The ADF model

The normalised CSD function of the ADF in the wavenumber-frequency space is given by [42].

$$
\tilde{\phi}_{p p}^{\mathrm{ADF}}\left(k_{x}, k_{y}, \omega\right)=\left\{\begin{array}{ll}
\frac{2 \pi}{k_{a} \sqrt{k_{a}^{2}-k_{x}^{2}-k_{y}^{2}},} & k_{a}^{2}>k_{x}^{2}+k_{y}^{2} \\
0, & k_{a}^{2} \leq k_{x}^{2}+k_{y}^{2}
\end{array}\right\},
$$

\section{References}

[1] M. B. S. Magalhães, R. A. Tenenbaum, Sound sources reconstruction techniques: A review of their evolution and new trends, Acta. Acust. united. Ac. 90 (2) (2004) 199-220.

[2] E. G. Williams, Supersonic acoustic intensity, J. Acoust. Soc. Am. 97 (1) (1995) 121-127.

[3] E. G. Williams, Supersonic acoustic intensity on planar sources, J. Acoust. Soc. Am. 104 (5) (1998) 2845-2850.

[4] E. Fernandez-Grande, F. Jacobsen, Q. Leclere, Direct formulation of the supersonic acoustic intensity in space domain, J. Acoust. Soc. Am. 131 (1) (2012) 186-193.

[5] E. Fernandez-Grande, F. Jacobsen, Conservation of power of the supersonic acoustic intensity, J. Acoust. Soc. Am. 136 (2) (2014) 461-465.

[6] N. P. Valdivia, E. G. Williams, P. C. Herdic, Equivalent sources method for supersonic intensity of arbitrarily shaped geometries, J. Sound. Vib. 347 (2015) 46-62.

[7] M. B. S. Magalhães, R. A. Tenenbaum, Supersonic acoustic intensity for arbitrarily shaped sources, Acta. Acust. united. Ac. 92 (2) (2006) 189-201.

[8] S. Marburg, E. Lösche, H. Peters, N. Kessissoglou, Surface contributions to radiated sound power, J. Acoust. Soc. Am. 133 (6) (2013) 3700-3705.

[9] E. G. Williams, Convolution formulations for non-negative intensity, J. Acoust. Soc. Am. 134 (2) (2013) 1055-1066. 
[10] C. C. Junior, R. Tenenbaum, Useful intensity: A technique to identify radiating regions on arbitrarily shaped surfaces, J. Sound. Vib. 332 (6) (2013) 1567-1584.

[11] V. Ferreira, R. Tenenbaum, F. Dias, C. Corrêa Jr, Power operator dimensional reduction to obtain the useful intensity in rectangular plates with several boundary conditions, J. Sound. Vib. 448 (2019) 130-145.

[12] D. Liu, H. Peters, S. Marburg, N. Kessissoglou, Surface contributions to scattered sound power using non-negative intensity, J. Acoust. Soc. Am. 140 (2) (2016) 1206-1217.

[13] D. Liu, H. Peters, S. Marburg, N. Kessissoglou, Supersonic intensity and non-negative intensity for prediction of radiated sound, J. Acoust. Soc. Am. 139 (5) (2016) 2797-2806.

[14] D. Liu, Z. Havránek, S. Marburg, H. Peters, N. Kessissoglou, Nonnegative intensity and back-calculated non-negative intensity for analysis of directional structure-borne sound, J. Acoust. Soc. Am. 142 (1) (2017) 117-123.

[15] D. Liu, S. Marburg, C. Geweth, N. Kessissoglou, Non-negative intensity for structures with inhomogeneous damping, J. Theor. Comput. Acoust. 27 (01) (2019) 1850050.

[16] D. R. Wilkes, H. Peters, P. Croaker, S. Marburg, A. J. Duncan, N. Kessissoglou, Non-negative intensity for coupled fluid-structure interaction problems using the fast multipole method, J. Acoust. Soc. Am. 141 (6) (2017) 4278-4288.

[17] M. Aucejo, N. Totaro, J.-L. Guyader, Identification of source velocities on 3D structures in non-anechoic environments: Theoretical background and experimental validation of the inverse patch transfer functions method, J. Sound. Vib. 329 (18) (2010) 3691-3708.

[18] D. Vigoureux, N. Totaro, J. Lagneaux, J.-L. Guyader, Inverse patch transfer functions method as a tool for source field identification, J. Vib. Acoust. 137 (2).

[19] N. P. Valdivia, Integral formulas for supersonic reconstruction of the acoustic field, Inverse. Probl. Eng. 26 (3) (2018) 376-397. 
[20] N. P. Valdivia, Improved integral formulae for supersonic reconstruction of the acoustic field, Inverse. Probl. Eng. 26 (6) (2018) 898-924.

[21] E. Ciappi, S. De Rosa, F. Franco, J. Guyader, S. Hambric, Flinovia - Flow Induced Noise and Vibration Issues and Aspects: A Focus on Measurement, Modeling, Simulation and Reproduction of the Flow Excitation and Flow Induced Response, EBL-Schweitzer, Springer International Publishing Switzerland, 2014.

[22] E. Ciappi, S. De Rosa, F. Franco, J. Guyader, S. Hambric, R. Leung, A. Hanford, Flinovia-Flow Induced Noise and Vibration Issues and Aspects-II: A Focus on Measurement, Modeling, Simulation and Reproduction of the Flow Excitation and Flow Induced Response, Springer International Publishing Switzerland, 2018.

[23] M. Karimi, P. Croaker, A. Skvortsov, D. Moreau, N. Kessissoglou, Numerical prediction of turbulent boundary layer noise from a sharp-edged flat plate, Int. J. Numer. Meth. Fl. 90 (2019) 522-543.

[24] E. G. Williams, Fourier acoustics: sound radiation and nearfield acoustical holography, Elsevier, San Diego, California, USA, 1999.

[25] C. Marchetto, Experimental characterization of the vibroacoustic response of panels under random excitations by sensitivity functions, Ph.D. thesis, Univ Lyon, INSA-Lyon, Laboratoire VibrationsAcoustique (LVA), France (2018).

[26] M. Karimi, L. Maxit, P. Croaker, O. Robin, A. Skvortsov, S. Marburg, N. Atalla, N. Kessissoglou, Analytical and numerical prediction of acoustic radiation from a panel under turbulent boundary layer excitation, J. Sound. Vib. (2020) 115372.

[27] A. Ghatak, S. Lokanathan, The Dirac Delta Function, Springer Netherlands, Dordrecht, 2004, pp. 3-18.

[28] X.-W. Gao, An effective method for numerical evaluation of general 2D and 3D high order singular boundary integrals, Comput. Methods in Appl. Mech. Eng. 199 (45-48) (2010) 2856-2864.

[29] K. M. Singh, M. Tanaka, Analytical integration of weakly singular integrals in boundary element analysis of Helmholtz and advection-diffusion 
equations, Comput. Methods in Appl. Mech. Eng. 189 (2) (2000) 625640.

[30] C. Maury, P. Gardonio, S. Elliott, A wavenumber approach to modelling the response of a randomly excited panel, Part I: General theory, J. Sound. Vib. 252 (1) (2002) 83-113.

[31] D. E. Newland, An introduction to random vibrations, spectral \& wavelet analysis, Courier Corporation, Mineola, New York, USA, 2012.

[32] M. Karimi, P. Croaker, L. Maxit, O. Robin, A. Skvortsov, S. Marburg, N. Kessissoglou, A hybrid numerical approach to predict the vibrational responses of panels excited by a turbulent boundary layer, J. Fluid. Struct. 92 (2020) 102814.

[33] L. Maxit, O. Guasch, V. Meyer, M. Karimi, Noise radiated from a periodically stiffened cylindrical shell excited by a turbulent boundary layer, J. Sound. Vib. 466 (2020) 115016.

[34] L. Maxit, M. Karimi, V. Meyer, N. Kessissoglou, Vibroacoustic responses of a heavy fluid loaded cylindrical shell excited by a turbulent boundary layer, J. Fluid. Struct. 92 (2020) 102758.

[35] L. Maxit, Simulation of the pressure field beneath a turbulent boundary layer using realizations of uncorrelated wall plane waves, J. Acoust. Soc. Am. 140 (2) (2016) 1268-1285.

[36] W. Graham, A comparison of models for the wavenumber-frequency spectrum of turbulent boundary layer pressures, J. Sound. Vib. 206 (4) (1997) 541-565.

[37] R. Mellen, Wave-vector filter analysis of turbulent flow, J. Acoust. Soc. Am. 95 (3) (1994) 1671-1673.

[38] M. Bull, Wall-pressure fluctuations associated with subsonic turbulent boundary layer flow, J. Fluid. Mech. 28 (4) (1967) 719-754.

[39] C. Marchetto, L. Maxit, O. Robin, A. Berry, Experimental prediction of the vibration response of panels under a turbulent boundary layer excitation from sensitivity functions, J. Acoust. Soc. Am. 143 (5) (2018) 2954-2964. 
541

542

[40] Z. Dingguo, M. J. Crocker, Sound power radiated from rectangular plates, Arch. Acoust. 34 (1) (2009) 25-39.

[41] G. Maidanik, Response of ribbed panels to reverberant acoustic fields, J. Acoust. Soc. Am. 34 (6) (1962) 809-826.

[42] C. Marchetto, L. Maxit, O. Robin, A. Berry, Vibroacoustic response of panels under diffuse acoustic field excitation from sensitivity functions and reciprocity principles, J. Acoust. Soc. Am. 141 (6) (2017) 4508-4521. 